\title{
ATRIAL FLUTTER AFTER LATERAL TUNNEL CONSTRUCTION IN THE MODIFIED FONTAN OPERATION: A CANINE MODEL
}

\author{
Mark D. Rodefeld, $\mathrm{MD}^{\mathrm{a} \S}$ \\ Burt I. Bromberg, $\mathrm{MD}^{\mathrm{b}}$ \\ Richard B. Schuessler, $\mathrm{PhD}^{\mathrm{a} s}$ \\ John P. Boineau, MD ${ }^{\mathrm{a}}$ \\ James L. Cox, MD \\ Charles B. Huddleston, $\mathrm{MD}^{\mathrm{a}}$
}

Intraatrial reentrant tachycardia, or atrial flutter, is a common postoperative problem after Fontan repair, which involves an atriopulmonary connection. A modification of Fontan repair, total cavopulmonary connection, minimizes the portion of the right atrium exposed to stretch and hypertension; however, atrial flutter continues to occur after this procedure. We postulated that the intraatrial lateral tunnel suture line of total cavopulmonary connection, in the absence of physiologic alterations such as atrial hypertension or stretch, provides the necessary electrophysiologic substrate for atrial flutter. The purpose of this study was to produce a canine model of total cavopulmonary connection (1) to establish that the intraatrial suture line alone is sufficient to permit sustained atrial flutter and (2) to characterize the pathways of resulting reentrant arrhythmias. After induction of general anesthesia, 25 to $30 \mathrm{~kg}$ dogs $(n=17)$ underwent median sternotomy, cradling of the pericardium, and placement of a pacing electrode on the right atrial appendage. Normothermic cardiopulmonary bypass was initiated. The total cavopulmonary connection suture line was placed through a standard right atriotomy, simulating construction of the lateral tunnel. After closure of the atriotomy, 253 point unipolar atrial endocardial form-fitting electrodes were placed through bilateral ventriculotomies. By means of atrial burst pacing and programmed extrastimulation, induction of atrial flutter was attempted. If atrial flutter could not be induced, isoproterenol was infused and the stimulation protocol was repeated. After induction of atrial flutter, mapping of the activation sequence was performed. Before suture line placement, no dog had inducible atrial flutter. After placement of the suture line, sustained atrial flutter was reproducibly induced in every dog, although isoproterenol was required for this in three $(\mathbf{1 7 . 6 \% )}$. The mean flutter cycle length was $177 \pm$ 30 msec. In each case, the atrial flutter circuit was limited to the right atrium, with the left atrium being passively activated. The atrial flutter circuit was dependent on a corridor of myocardium that resulted from conduction block on the free wall, created by the lateral margin of the total cavopulmonary connection. In no case was the atriotomy integral to the atrial flutter circuit. This study establishes that the total cavopulmonary connection baffle suture line alone, without alteration in circulatory
From the Division of Cardiothoracic Surgery, Department of Surgeiy, ${ }^{a}$ and Division of Pediatric Cardiology, Department of Pediatrics, ${ }^{\mathrm{b}}$ Washington University School of Medicine, St. Louis, Mo.

Supported by National Institutes of Health grants HL32257 and HL33722. Dr. Rodefeld was supported by a National Research Service Award Grant (HL08894).

Read at the Seventy-fifth Annual Meeting of The American Association for Thoracic Surgery, Boston, Mass., April 23-26, 1995.
Address for reprints: Charles B. Huddleston, MD, Department of Surgery, Division of Cardiothoracic Surgery, Box 8234, 3308 Queeny Tower, One Barnes Hospital Plaza, St. Louis, MO 63110 .

J Thorac Cardovasc Surg 1996;111:514-26

${ }^{\S}$ By invitation.

Copyright (C) 1996 by Mosby-Year Book, Inc.

$0022-5223 / 96 \$ 5.00+0 \quad \mathbf{1 2 / 6 / 6 9 9 5 2}$ 


\begin{abstract}
physiology, creates a sufficient anatomic substrate for atrial flutter in a short-term canine model. Delineation of the anatomic boundaries of the reentrant circuit raises the possibility of targeting areas within the circuit that could be modified, potentially reducing the incidence of postoperative atrial flutter after total cavopulmonary connection. (J THORAC CARDIOvaSC SURG 1996;111:514-26)
\end{abstract}

Intraatrial reentrant tachycardia, or atrial flutter (AFL), results in substantial morbidity among patients who have undergone the Fontan operation. ${ }^{1-3}$ Supraventricular tachycardia is poorly tolerated by patients with single-ventricle physiology. Pharmacologic treatment is often ineffective and may be complicated by the proarrhythmic and negative inotropic properties of antiarrhythmic drugs.

Clinical studies suggest that right atrial hypertension may be a risk factor for both early and late arrhythmias after classic Fontan repair. ${ }^{4}$ This operation uses an atriopulmonary connection that leads to extreme dilatation as well as to elevated pressure in the entire right atrium. Total cavopulmonary connection (TCPC) is a recent modification of the Fontan procedure in which a C-shaped lateral tunnel, or baffle, is used to direct blood from the inferior vena cava through the right atrium to the pulmonary artery. ${ }^{5}$ It was anticipated that the TCPC would reduce the incidence of postoperative AFL because only a small portion of the right atrium is exposed to stretch and hypertension. ${ }^{6}$ Intermediate follow-up to 5 years, however, indicates that tachyarrhythmias after the TCPC continue to be a problem, ranging in frequency from $4 \%$ to $35 \% .^{4,7,8} \mathrm{We}$ therefore hypothesized that the lateral tunnel suture line alone, in the absence of changes in circulatory physiology, establishes an anatomic substrate for AFL. The objectives of this study were as follows: (1) to determine whether the lateral tunnel suture line alone is sufficient to permit sustained AFL and (2) to characterize the pathways of resultant arrhythmias.

\section{Methods}

Operation. Adult mongrel dogs weighing 25 to $30 \mathrm{~kg}$ $(n=17)$ were anesthetized with intravenous pentobarbital sodium $(30 \mathrm{mg} / \mathrm{kg})$, intubated with a cuffed endotracheal tube, and mechanically ventilated with a volume-cycled ventilator. An adequate level of anesthesia was maintained by intermittent infusion of 1 to $2 \mathrm{mg}$ pentobarbital sodium. Limb-lead electrocardiography was monitored, and a 20-gauge arterial pressure catheter was placed in the left femoral artery. After median sternotomy, the azygous vein was ligated and the heart cradled in the pericardium.
Bipolar pacing and sensing electrodes were sutured to the right and left atrial appendages, respectively. After systemic heparinization $(1 \mathrm{mg} / \mathrm{kg})$, a $16 \mathrm{~F}$ arterial cannula was placed into the right femoral artery and bicaval venous cannulation was performed with $28 \mathrm{~F}$ venous cannulas positioned as far as possible away from the right atrium. Normothermic cardiopulmonary bypass was instituted; to avoid acute changes in postoperative atrial refractoriness, cardioplegic arrest was not used. A standard longitudinal right atriotomy was made from the midatrial appendage, anterior to the crista terminalis, to the level of the sinus node artery in the lower right atrial free wall. Care was taken to avoid disruption of the sinus node artery, which arises from the distal right coronary artery in $95 \%$ of dogs.

A continuous 4-0 polypropylene suture was then placed inside the right atrium in a manner simulating lateral tunnel construction in TCPC; no baffle was placed. The suture line started at the limbus of the fossa ovalis and traveled inferiorly, lateral to the fossa ovalis, between the coronary sinus and the inferior vena caval orifice, around the inferior vena cava, laterally up the crista terminalis, around the superior vena caval orifice, and back to the limbus of the fossa ovalis (Fig. 1, $A$ ). The atriotomy was then closed with a continuous 4-0 polypropylene suture.

Electrophysiology testing. Bilateral ventriculotomies were made for insertion of two form-fitting silicone elastomer (Dow Corning Corp., Midland, Mich.) atrial endocardial multipoint electrodes containing 253 unipolar unit electrodes. Electrode surface templates were constructed from plaster casts molded from postmortem formalinfixed canine atria to fit the complex endocardial contours of each atrium. Interelectrode distance between unipolar points was 6 to $8 \mathrm{~mm}$. After excision of mitral and tricuspid valve leaflets, electrodes were positioned and anchored with several interrupted 4-0 silk sutures at the anulus. Placement was confirmed by palpation through the atrial myocardium. An indifferent electrode was attached to the chest wall for a unipolar reference (Fig. 1, $B$ ).

Atrial burst pacing at cycle lengths of 200 to $400 \mathrm{msec}$ was performed before and after the atriotomy. After the TCPC suture line was placed and the intraatrial electrodes were implanted, the cycle length of the atrial burst pacing was shortened to 110 to $250 \mathrm{~ms}$. In six dogs, single atrial premature stimuli were delivered after an 8-beat drive train of 200 to $250 \mathrm{msec}$. When sustained AFL could not be induced, a $0.3 \mu \mathrm{g} / \mathrm{kg}$ bolus of isoproterenol was injected into the cardiopulmonary bypass circuit and the pacing protocol was repeated.

Sustained AFL was defined as a fixed cycle length tachycardia of greater than 30 seconds in duration. Reproducibility was determined by reinduction of the tachy- 

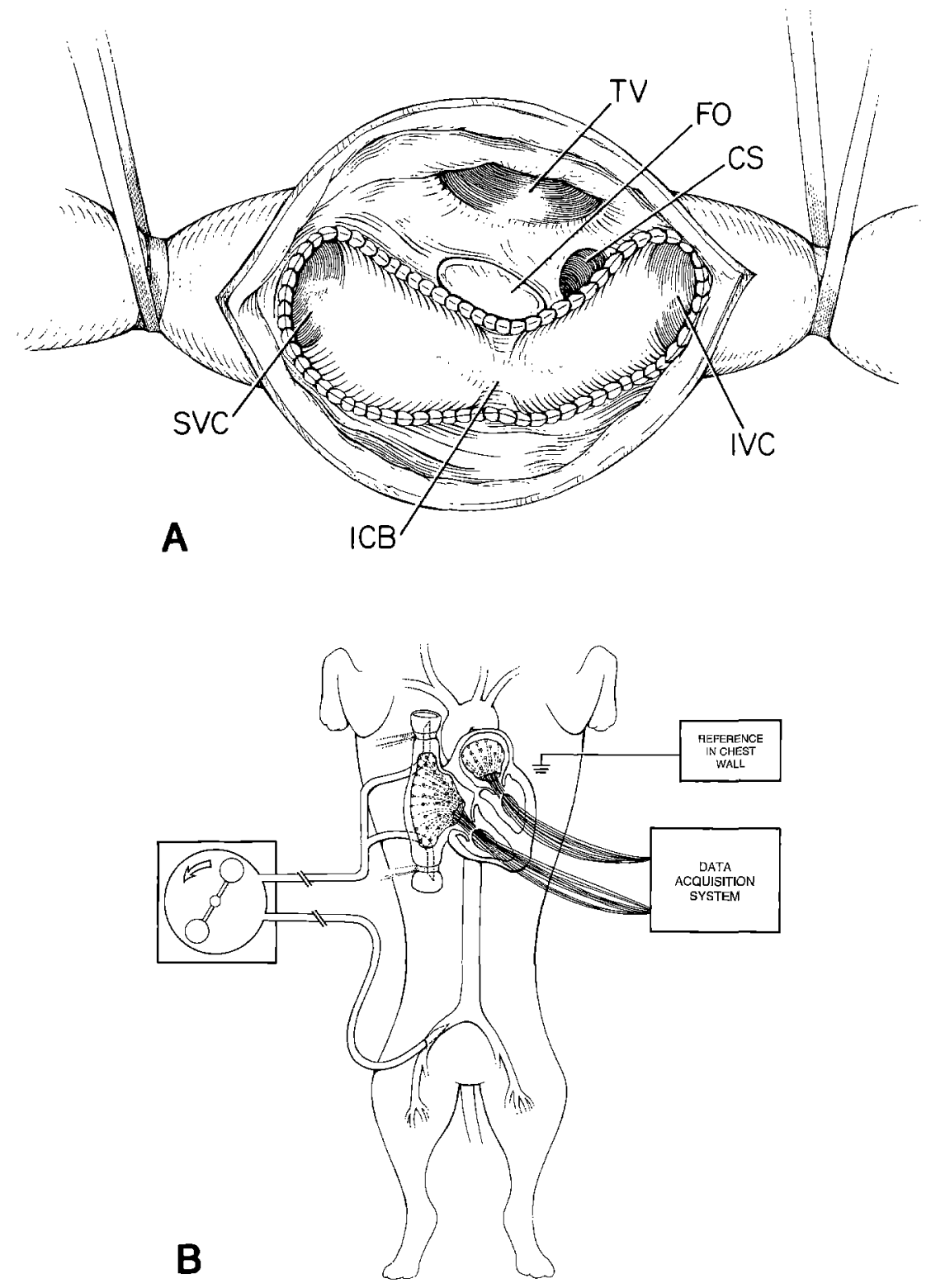

Fig. 1. A, Surgeon's view of the lateral tunnel suture line of the TCPC. The TCPC suture line is placed starting at the medial intercaval band $(I C B)$ at the limbus of the fossa ovalis $(F O)$, runs inferiorly lateral to the FO, between the coronary sinus (CS) os and the inferior vena cava $(I V C)$, around the IVC, superiorly up the crista terminalis to the superior vena cava $(S V C)$, around the SVC orifice, and back to the limbus of the FO. $T V$, Tricuspid valve. B, Laboratory setup. Form-fitting atrial endocardial multipoint electrodes containing 253 unipolar unit electrodes are inserted through bilateral ventriculotomies into the left and right atria.

arrhythmia with the same extrastimulus pattern that had originally induced it.

Data acquisition and analysis. Atrial activation sequence data were obtained by simultaneously recording 253 unipolar electrograms from the endocardial multipoint electrodes. Limb-lead electrocardiograms and bipolar atrial electrograms from either the right or left atrial appendage were also simultaneously recorded. Data were recorded during spontaneous rhythm and during any sustained arrhythmia with a 256-channel computerized data-acquisition and data-analysis system based on a VaxStation II/GPX graphics workstation connected to two 128-channel PDP 11/23+-based data-acquisition subsystems (Digital Equipment Corporation, Maynard, Mass.). The system is run with in house-developed software (GLAS) for data acquisition control, management, 
display, and analysis. Unipolar electrograms were recorded at a gain of 1000 with a frequency response of 50 to $500 \mathrm{~Hz}$. Each channel was digitized at $1000 \mathrm{~Hz}$ with a 12-bit resolution. Local endocardial activation times were determined from the time of the maximum negative derivative $(-\mathrm{dV} / \mathrm{dt})$ of the unipolar electrogram. All electrograms were edited visually to verify accuracy of computer-selected activation times. Computer-generated activation sequence maps were reconstructed from all recordings. Data processing and three-dimensional interactive display was performed on a Silicon Graphics Iris 4D/320GTX high-performance graphic workstation (Silicon Graphics Inc., Mountain View, Calif.). Activation sequence maps were displayed as real-time three-dimensional movie images on a three-dimensional surface model of the canine atrium. ${ }^{9}$

All animals received humane care in compliance with the "Principles of Laboratory Animal Care" formulated by the National Society for Medical Research and the "Guide for the Care and Use of Laboratory Animals" prepared by the National Academy of Science and published by the National Institutes of Health (NIH Publication 86-23, revised 1985). In addition, the study protocol was approved by the Washington University Animal Studies Committee.

\section{Results}

AFL could not be induced in any case either before or after atriotomy alone. Although rapid atrial burst pacing was often required to induce AFL after placement of the lateral tunnel suture line, when done before or after atriotomy alone paced cycle lengths less than $150 \mathrm{msec}$ typically induced either atrial fibrillation or an extremely rapid tachycardia (cycle length $\leq 120 \mathrm{msec}$ ). Consequently, to minimize the need for direct current cardioversion, atrial burst pacing at cycle lengths less than 200 msec was not attempted in later studies until after the suture line was in place.

AFL was reproducibly induced in 14 of 17 animals $(82 \%)$ after placement of the lateral tunnel suture line (Table I), with a mean cycle length of $173 \pm 30$ msec. In the three remaining dogs, after isoproterenol was administered AFL was induced with a mean cycle length of $194 \pm 33 \mathrm{msec}$ (Table I). The tachycardias induced met the conventional clinical criteria for AFL. They could be pace induced and pace terminated, and they exhibited a fixed cycle length that was not dependent on participation of the ventricle (Fig. 2). The presence of reentry within the atrium was confirmed by atrial activation sequence maps, which demonstrated continuous electrical activity throughout the entire tachycardia cycle length (Fig. 3).

The circuit of the AFL observed in each experiment was limited entirely to the right atrium, with
Table I. $A F L$ cycle length and direction

\begin{tabular}{cccc}
\hline Study & $C L($ msec $)$ & Direction & Pathway \\
\hline 1 & 177 & + & 1 \\
2 & 130 & + & 2 \\
3 & 220 & + & 1 \\
4 & 130 & - & 2 \\
5 & 160 & - & 1 \\
6 & 210 & - & 1 \\
7 & 166 & + & 2 \\
8 & 179 & - & 1 \\
9 & 146 & - & 1 \\
10 & 196 & + & 1 \\
11 & 205 & + & 1 \\
12 & $188^{*}$ & - & 1 \\
13 & $165^{*}$ & + & 1 \\
14 & $230^{*}$ & - & 1 \\
15 & 194 & - & 1 \\
16 & 180 & + & 2 \\
17 & 138 & & 1 \\
\hline
\end{tabular}

Cycle length $177 \pm 31$ msec (mean \pm standard error of the mean), $n=17$. Pathway 1 signifies reentrant circuit rotation around both venae cavae, pathway 2 signifies circuit breakthrough at the lateral aspect of the lateral tunnel suture line. $C L$, Cycle length; + , clockwise rotation; -, counterclockwise rotation.

*Administration of isoproterenol.

passive activation of the left atrium (Fig. 3). The atriotomy and the septal portion of the lateral tunnel suture line had no impact on wave front propagation. In contrast, complete block occurred along the free wall segment of the lateral tunnel suture line, corresponding to the site of the crista terminalis. This line of conduction block created an isthmus of myocardium between that portion of the suture line on the free wall and the tricuspid anulus. Were it not for the conduction block along the crista terminalis, competing wave fronts from the posterior lateral free wall would have entered this isthmus between the suture line and the tricuspid anulus prematurely. Although the conduction velocity, illustrated by the distance between $10 \mathrm{msec}$ isochrones, was slower on the free wall than on the septum, there were no discrete regions of slow conduction within the circuit that were integral to maintaining the tachycardia. The wave front traveled through the isthmus on the free wall at a uniform velocity and then swept onto the septal surface, where it straddled the suture line, moving rapidly in a caudal direction. Conduction was also not affected by the atriotomy site.

The line of block on the free wall was anatomic rather than functional, as evidenced by its persistence during sinus rhythm (Fig. 4). Earliest activation occurred in the intercaval region of the free wall, near the sinus node. As the wave front propa- 

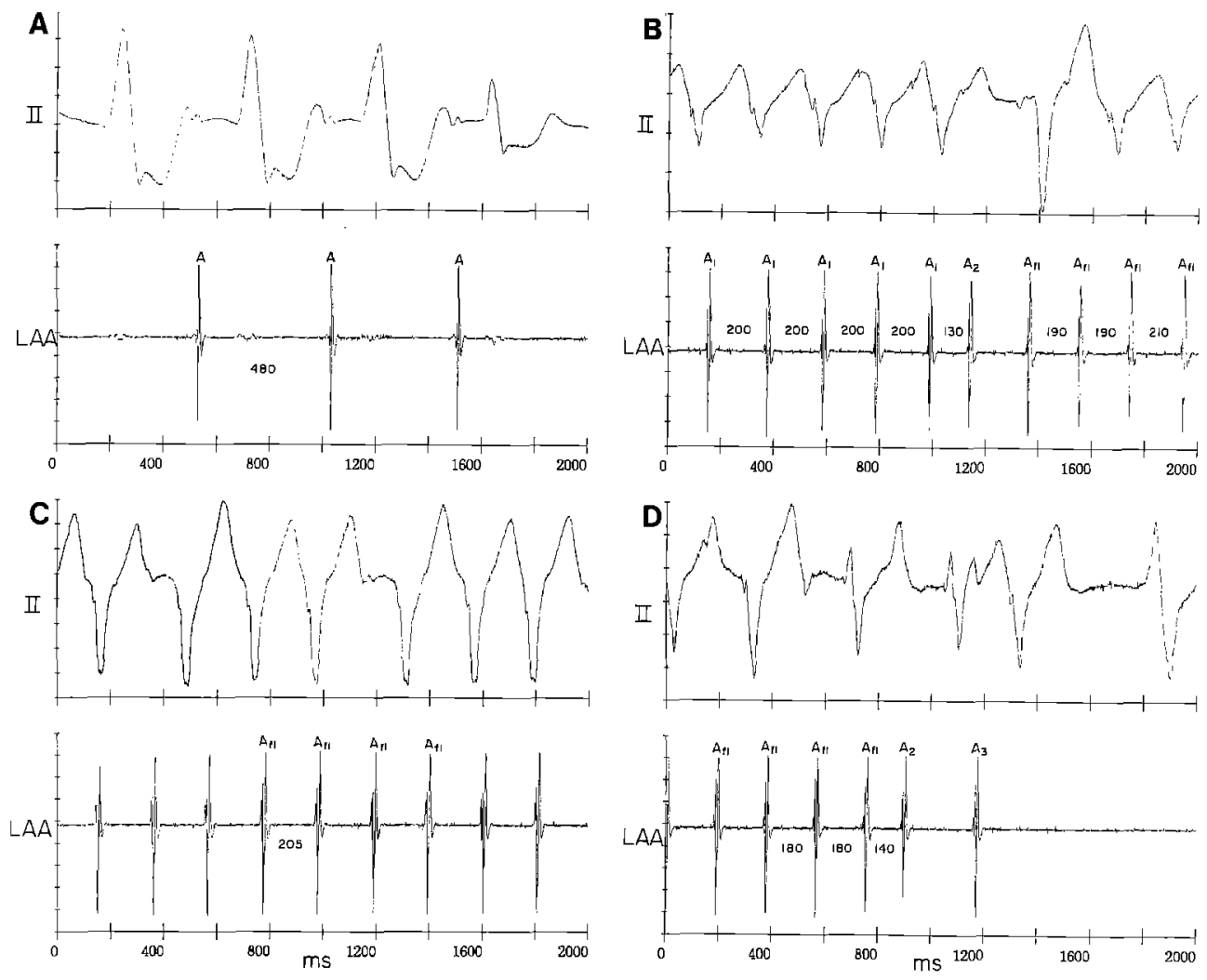

Fig. 2. Initiation and termination of AFL. In each panel, lead II is displayed in the upper trace and a bipolar electrogram from the left atrial appendage $(L A A)$ is displayed in the lower trace. All recordings are taken from the same study. A, Sinus rhythm after Fontan suture line placement (cycle length $480 \mathrm{msec}$ ). The bundle-branch block observed in the QRS complex resulted from bilateral ventriculotomies required for insertion of biatrial endocardial electrodes. B, AFL induced by programmed extrastimulation from the right atrial appendage. After a drive train at a cycle length of $200 \mathrm{msec}$, a single premature beat initiates the tachycardia (cycle length $190 \mathrm{msec}$ ). C, Tachycardia exhibits a stable cycle length of $205 \mathrm{msec}$. Surface lead in the upper trace demonstrates atrioventricular nodal Wenckebach, proving that the ventricle is not a participant in the tachycardia. $\mathbf{D}$, AFL is terminated with a single atrial premature beat. The next beat reflects either a spontaneous beat or an echo, followed by a long pause as the sinus node resets. $A$, Spontaneous sinus rhythm, $A_{1}$, paced beats during atrial drive train, $A_{2}$, paced premature beat, $A_{3}$, spontaneous beat or echo beat, $A_{f}$, AFL.

gated from the region of the sinus node, it encountered a zone of block corresponding to the site of the suture line along the crista terminalis. In the example shown, the block was not complete; time isochrones demonstrate a breakthrough in the rostral section. The block is sufficient, however, to result in delayed activation of the corridor between the suture line and the tricuspid anulus. This corridor is activated by wave fronts that travel posteriorly around the inferior and superior venae cavae, where they cross over the suture lines and return onto the free wall. As it is during AFL, conduction along the septum is rapid, uniform, and unaltered by the lateral tunnel suture line.

Multiple potential circuits within the right atrium were observed, but all were dependent on the line of block on the free wall that created an isthmus of myocardium between it and the tricuspid anulus. Pathways traveled in both clockwise and counterclockwise loops as viewed from the tricuspid anulus (Figs. 3 and 5), illustrating the potential for at least two different circuits in every dog. Another mechanism for multiple circuits was observed in two dogs in which the line of block along the free wall 

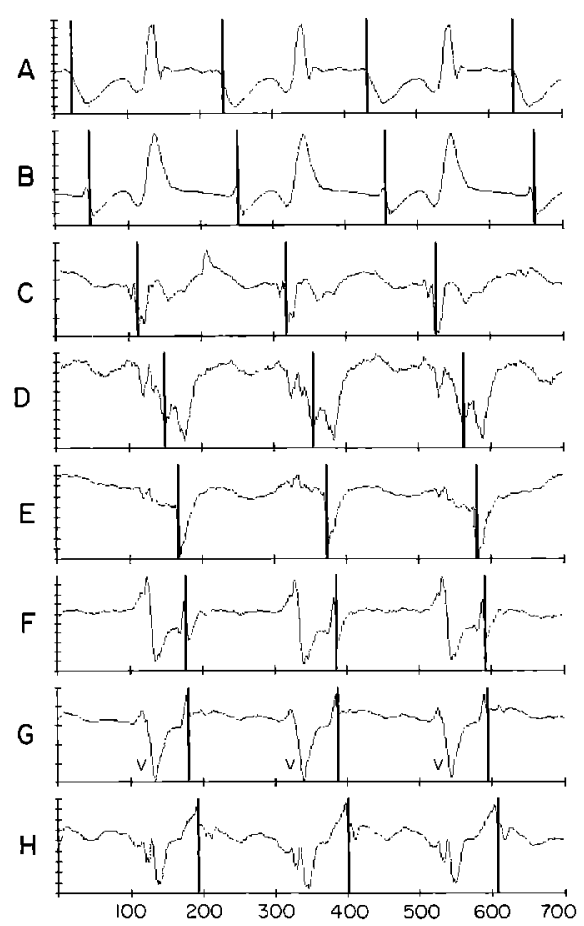
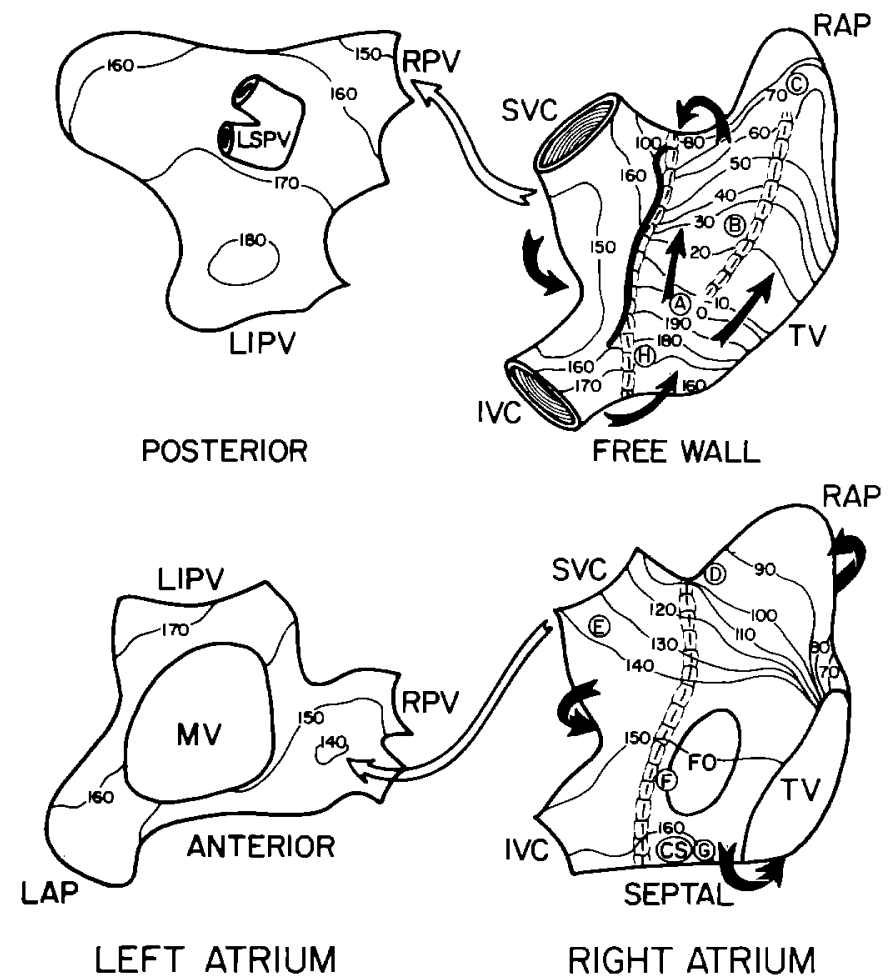

Fig. 3. Activation sequence during AFL. Electrograms on the left were taken from serial sites represented by corresponding letters in the diagrams on the right. Left, Electrograms from multiple sites in the right atrium demonstrate continuous electrical activity. Right, The free wall and posterior surfaces of the right and left atria are represented in the upper diagrams, and the septal and anterior surfaces are represented in lower diagrams. Atriotomy and lateral tunnel suture lines are represented by broken hatched lines. Time isochrones show the activation sequence in right and left atria. Solid arrows represent the direction of wave front propagation, which is clockwise viewed from the tricuspid anulus. Beginning at site $A$, the wave front travels rostrally up the free wall (site $B$ ) before advancing around that atrial appendage and tricuspid anulus in a clockwise direction onto the septal surface (site $D$ ). Moving down the septum on both sides of the suture line (sites $E$ and $F$ ), the wave front crosses the floor of the right atrium (site $G$ ), arriving back on the septal surface (site $H$ ) to complete the circuit. $R A P$, Right atrial appendage; $R P V$, right pulmonary vein; $S V C$, superior vena cava; $L S P V$, left superior pulmonary vein; $L I P V$, left inferior pulmonary vein; $T V$, tricuspid valve; $I V C$, inferior vena cava; $M V$, mitral valve; $F O$, fossa ovalis; $L A P$, left atrial appendage; $C S$, coronary sinus.

segment of the suture line was incomplete (Fig. 5). This allowed the reentrant impulse to cross over the suture line on the caudal margin of the free wall, in contrast to the more typical circuit in which the wave front exited the free wall corridor by traversing the floor of the right atrium between the tricuspid anulus and the inferior vena cava. As conduction proceeded down the free wall anterior to the suture line, it broke across the suture line and back onto the septum by propagating around either the inferior or the superior vena cava. In the absence of breakthrough along the free wall component of the suture line, the circuit presumably would have been completed, as shown in Fig. 3, by traveling along the floor of the atrium.

Although the conduction block created by the lateral tunnel suture line created the substrate for AFL, it alone did not account for how the tachycardia was triggered. Classically, macroreentry requires unidirectional conduction to initiate the tachycardia. Activation sequence maps during programmed extrastimulation demonstrated that this requisite unidirectional conduction resulted from functional block (Fig. 6). In the example shown, pacing from the right atrial appendage during the drive train at a cycle length of $200 \mathrm{msec}$ resulted in uniform con- 


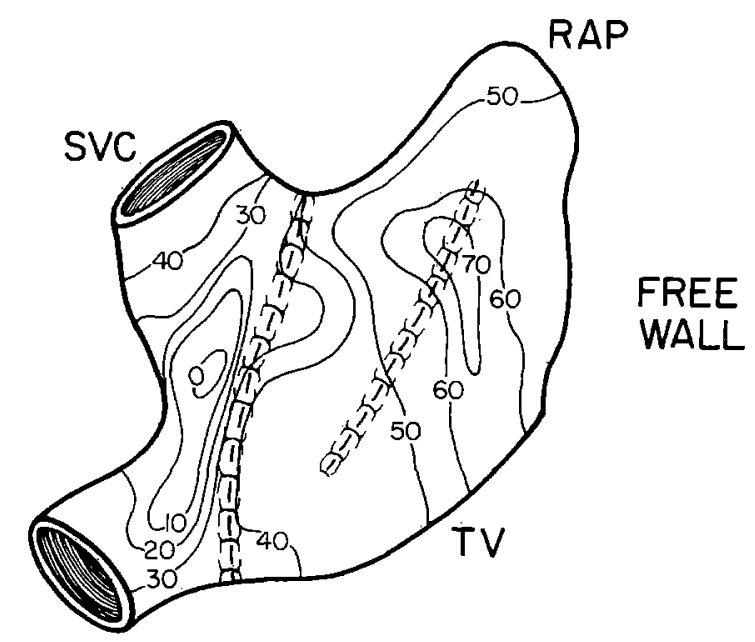

RIGHT ATRIUM

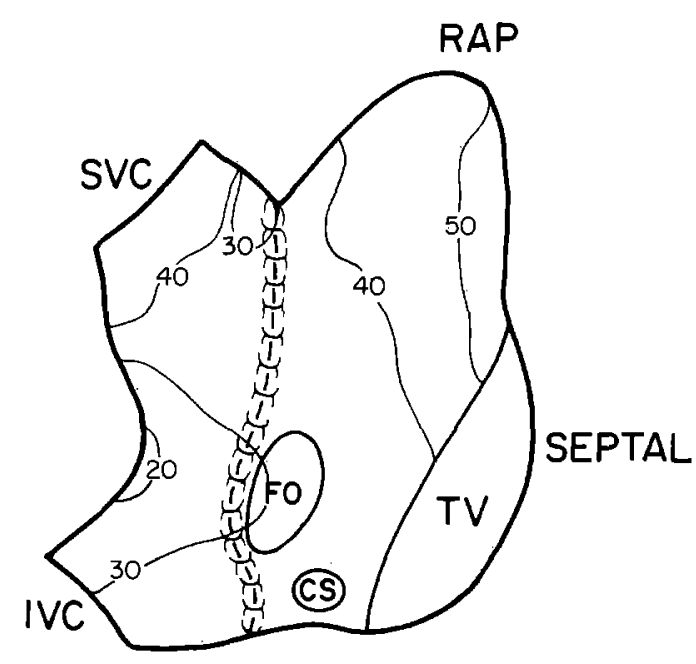

Fig. 4. Activation sequence during sinus rhythm. The free wall of the right atrium is displayed in the upper panel; the septal surface is displayed in the lower panel. Lateral tunnel and atriotomy suture lines are represented by broken hatched lines as in Fig. 3. Time isochrones obtained during sinus rhythm show conduction block along the free wall where the lateral tunnel suture line has been placed. There is no alteration in conduction resulting from the atriotomy. $R A P$, right atrial appendage; $S V C$, superior vena cava; $T V$, tricuspid valve. $F O$, fossa ovalis; $I V C$, inferior vena cava; $C S$, coronary sinus; $L I P V$, left inferior pulmonary vein; $L S P V$, left superior pulmonary vein; $M V$, mitral valve; $R P V$, right pulmonary vein.

duction down both the septum and the free wall. With a premature beat at the end of the drive train $\left(A_{1} A_{2} 130 \mathrm{msec}\right)$, however, the wave front encountered functional block on the free wall unrelated to any surgical or naturally occurring barriers. The block was considered to be functional on the basis of the conduction of preceding beats of the drive train, which were at a longer cycle length. During the drive cycle, the paced impulse produced two wave fronts, one propagating down the septum and the other down the free wall. These impulses normally collide and are extinguished. On the premature beat shown in Fig. 6, however, only the septal wave front propagated. When it reached the free wall it was unopposed by the second wave front, which had encountered a region of functional block. Because the functional block on the free wall was unidirectional, the single wave front continued its rostral course, initiating the tachycardia.

\section{Discussion}

Despite initial optimism that TCPC would lessen the incidence of postoperative atrial tachyarrhythmias in patients with single ventricle physiology, such a reduction has not consistently been observed. Even in the most favorable studies, the incidence of atrial tachyarrhythmias remains $4 \%{ }^{8}$ Furthermore, follow-up periods have been short, less thä 5 years. Extrapolating from the experience after operations for other types of congenital heart disease, particuIarly Mustard repair for transposition of the great arteries, the incidence of atrial arrhythmias may be expected to increase for years after the operation. ${ }^{10}$

Minimum substrate for AFL. The determinants of human AFL after TCPC are poorly understood. Clinical studies have implicated functional block in human $\mathrm{AFL},{ }^{11,12}$ and a recent study demonstrated functional block related to the crista terminalis and eustachian ridge. ${ }^{13}$ Animal models of AFL have demonstrated functional components that may be related to a variety of factors, including atrial distention and fibrosis. ${ }^{14,15}$ Atrial hypertension has also been implicated in human beings. ${ }^{4} 16$ The results of this study demonstrate that in the short term, however, the anatomic barrier imposed by the suture line alone in TCPC is sufficient to permit sustained AFL. The study design avoided alterations in atrial physiology inherent in the TCPC operation by using a suture line without the baffle, eliminating the confounding variables of atrial stretch and hypertension.

The segment of the TCPC line on the free wall consistently produced a line of block during both sinus rhythm and AFL. The isthmus of myocardium between this surgical line of block and the naturally occurring barrier of the tricuspid anulus was critical to perpetuating the tachycardia. The suture line on 
A

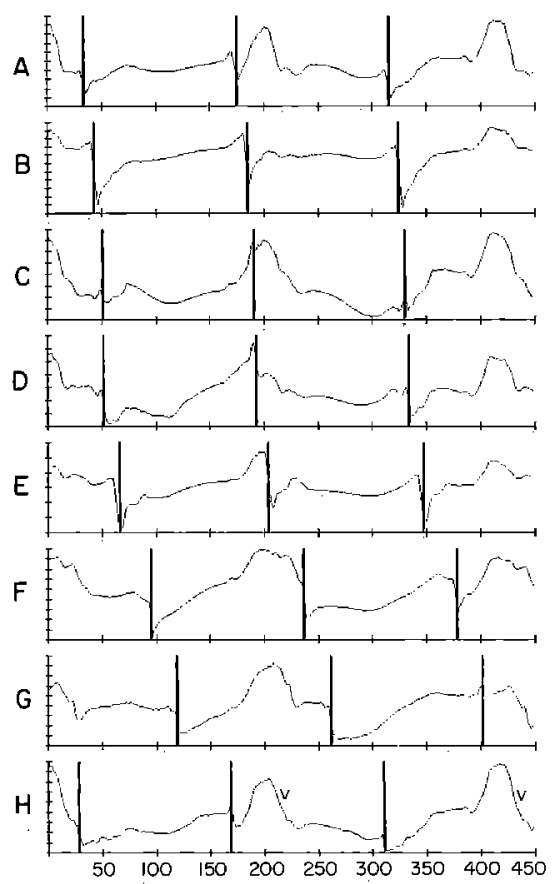

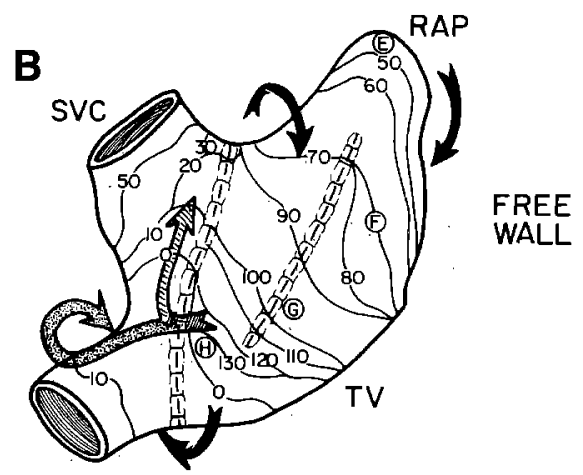

RIGHT ATRIUM

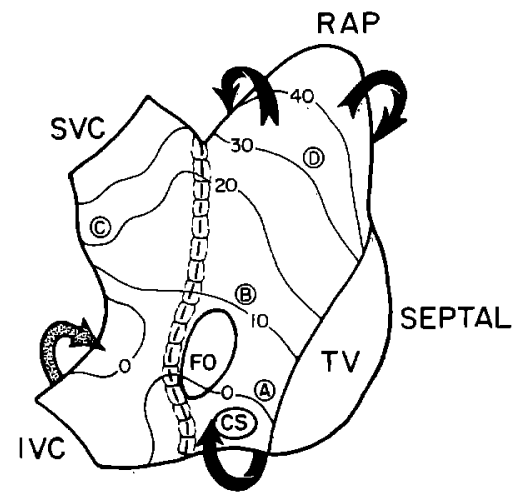

Fig. 5. Multiple AFL circuits. A, Electrograms lettered $A$ through $H$ correspond with sites denoted in the adjacent diagram and show continuous electrical activity through the AFL cycle length. B, The free wall and septum of the right atrium are displayed with suture lines as in previous figures. Time isochrones again show the activation sequence. Beginning at site $A$ on the septal surface, the wave front moves rostrally (sites $B, C$, and $D$ ), advancing around the atrial appendage and tricuspid anulus in a counterclockwise direction. After traveling caudally on the free wall (sites $E, F$, and $G$ ), the wave front crosses over the lateral tunnel suture line (site H). Stippled arrow and hatched arrow demonstrate two potential routes of wave front propagation from this crossover point. It was not possible to determine in this dog which of these routes was responsible for completion of the reentry circuit. Solid arrow demonstrates the course by which the wave front most commonly reaches the septum. Presumably, this would have been the route by which the tachycardia circuit was completed in the absence of the crossover point on the free wall. Abbreviations as in other figures.

the free wall protected this isthmus from competing wave fronts in a manner similar to the $\mathrm{Y}$-incision described by Frame and coworkers. ${ }^{17,} 18$ No pathologic zones of slow conduction were required. Viewed differently, the line of block prevented the wave front within this zone from exiting at sites other than at the caudal or rostral margins of the free wall. This created a sufficiently long reentry circuit that the myocytes within this zone were once again excitable by the time the wave front exited one end of this corridor and returned to the other end.

In contrast to the portion of the suture line on the free wall, the TCPC segment of the suture line on the septal side did not appear to affect conduction. This may be related to anatomic differences in the two surfaces. The suture line on the free wall was created with full-thickness bites, whereas those on the septal surface were not. Previous studies from this laboratory have demonstrated that the right and left sides of the atrial septum are functionally and anatomically separate over most of their distribution. ${ }^{19}$ There are discrete regions of communicating myocyte bundles, however, which may have provided a mechanism by which impulses traveled along the septum in a uniform fashion despite the sutures. Impulses could have crossed over to the left side of 


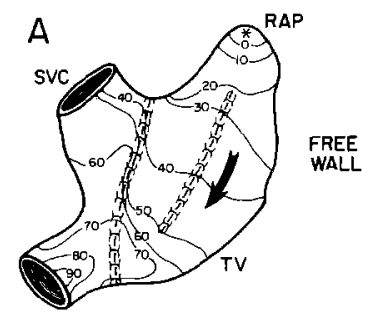

RIGHT ATRIUM

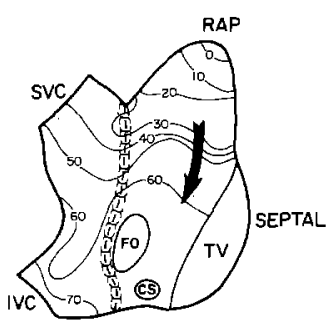

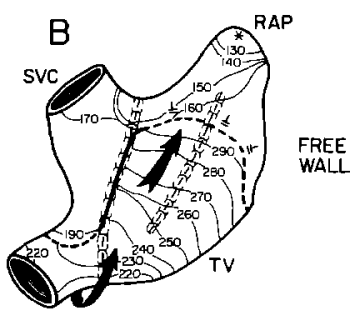

RIGHT ATRIUM

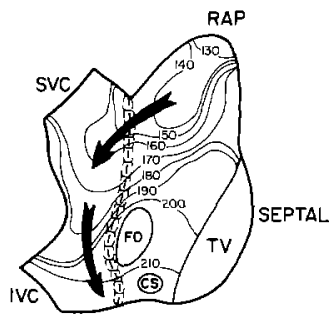

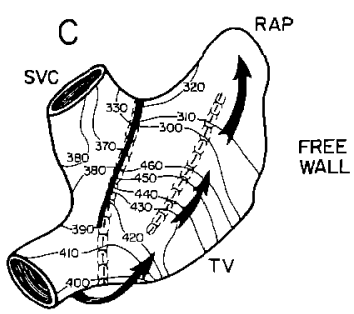

RIGHT ATRIUM

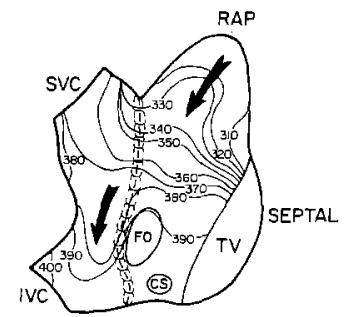

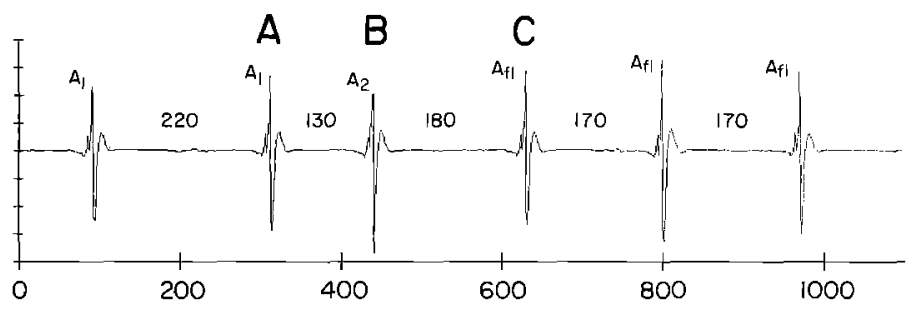

Fig. 6. Initiation of AFL. Free wall and septal surfaces in $\mathbf{A}, \mathbf{B}$, and $\mathbf{C}$ are displayed as in previous figures. Abbreviations are as in previous figures. The tracing below shows an atrial electrogram during initiation of AFL with a premature beat delivered after an 8 beat drive train. Activation sequences of $A_{1}, A_{2}$, and $A_{f}$ are shown in $\mathbf{A}, \mathbf{B}$, and $\mathbf{C}$, respectively. A, Atrial conduction after pacing from the right atrial appendage proceeds evenly down the free wall and septum. B, Conduction of the premature beat blocks on the free wall (illustrated by dotted horizontal line). This functional block leads to unidirectional conduction as the impulse propagates caudally down the septum and then rostrally onto the free wall. C, AFL perpetuates in a clockwise loop viewed from the tricuspid anulus.

the septum at the caudal margin, propagated up the unaffected left atrial component of the septum, and then crossed again to the right atrial component.

Potential for multiple reentry circuits. One of the puzzling aspects of clinical AFL in patients with Fontan physiology is the presence of apparent multiple reentry circuits, as determined by variable cycle lengths and differing $\mathrm{P}$-wave morphologic patterns on the surface electrocardiogram. It is not uncommon in patients with AFL to see two or three different cycle lengths, each with a different P-wave morphologic pattern. The findings from this model suggest two mechanisms by which this may happen. Clockwise and counterclockwise loops were equally prevalent in the dogs studied. Although we did not observe examples of circus movement tachycardia in opposite directions in the same dog, it seems likely that this would occur clinically. This would result in different $\mathrm{P}$-wave morphologic patterns and, depending on whether conduction velocity is affected by the direction of the wave front, possibly a change in cycle length. A second way that this might happen is illustrated by the dog who exhibited at least three different routes by which the impulse could rotate around the suture line on the free wall (Fig. 5). Each route would presumably produce a different cycle length and a different $\mathrm{P}$-wave morphologic pattern. Regardless of the potential for multiple circuits in any single dog, however, the AFL in all cases had in common a dependence of the protected isthmus on the free wall created by the lateral tunnel suture line.

Role of functional block. Macroreentrant tachycardias such as AFL require not only an electrophysiologic substrate but a trigger as well. One of the characteristics of atrial tachyarrhythmias after 
Fontan repair is the progressive increase in frequency with time. If the electrophysiologic substrate for AFL is an anatomic barrier present from the time of operation, other factors must be operative to explain late onset. One factor that might change with time and thus further predispose a patient toward the development of AFL is the extent of functional block. In an example of AFL initiated by an atrial premature beat, unidirectional block was responsible for initiating the tachycardia (Fig. 6). This unidirectional block was functional, occurring in a portion of the atrium unrelated to surgical or naturally occurring barriers. There are multiple potential explanations for increasing functional block with time, including diminished conduction safety margin, changes in gap junction function, and alterations in autonomic tone. Perhaps the most likely cause of functional block in this setting is an increase in dispersion of refractoriness. For example, progressive fibrosis may increase the probability of functional unidirectional block. If the frequency of atrial premature beats rises with time, the probability of an ill-timed event that initiates reentry is further increased. Alterations in the trigger for AFL may therefore account for the clinically observed late onset of arrhythmias.

Comparison of TCPC with previous models of AFL. Previous surgical models of AFL share common features with our TCPC model. In the classic study of Rosenblueth and Garcia Ramos, ${ }^{20}$ a crush injury in the intercaval region resulted in sustained and reproducible AFL circulating around both caval orifices (Fig. 7, A). Subsequent animal models of AFL have supported these findings. ${ }^{21,22}$ More recently, Frame and coworkers ${ }^{17,18}$ described a modification of the intercaval crush model in which a Y-shaped spur incision was extended from the intercaval lesion to the right atrial appendage (Fig. 7, $B$ ). The spur incision forced the reentrant circuit to rotate instead around the tricuspid anulus. A canine model of AFL after Mustard repair showed a similar circuit involving the tricuspid anulus, although the role of anatomic barriers in this operation is much more complicated than in other models. ${ }^{23}$

In this study, the activation sequence maps of AFL could be interpreted as consistent with conduction either around the caval orifices, as in the model of Rosenbleuth and Garcia Ramos, ${ }^{20}$ or around the tricuspid anulus, as in the model of Frame and coworkers $^{17,18}$ (Fig. 7, C). In reality, the reentry circuit is not a line, as it is often portrayed in schematic diagrams, but rather a band of tissue. In
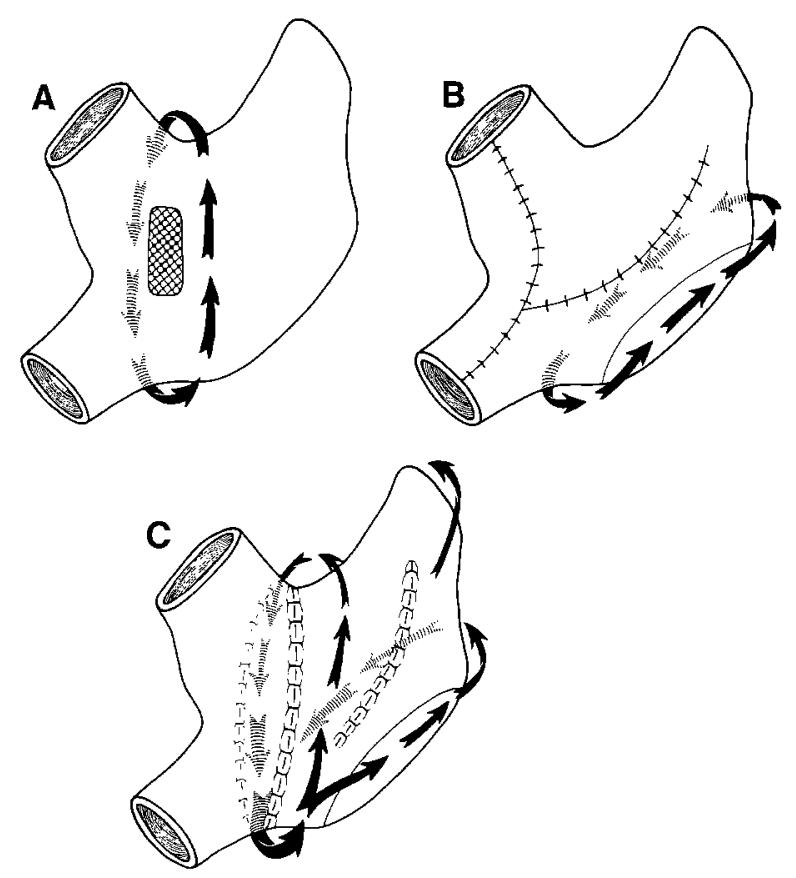

Fig 7. Schematic illustrations depicting reentrant pathways in different canine models of AFL. A, The classic model of Rosenblueth and Garcia Ramos, ${ }^{20}$ in which an intercaval crush injury results in reentry around the caval orifices. B, Model of Frame and coworkers, ${ }^{18}$ in which the Rosenblueth model was modified to include a Y-shaped spur incision extending from the intercaval incision to the midportion of the right atrial appendage. $\mathbf{C}$, Predominant pathway observed in the series reported here. The reentrant pathway exists between the two obstacles demonstrated in the other two models. At the low right atrium (wide arrow), the borders of conduction are closely related between the tricuspid anulus and the inferior vena cava, resulting in focal conduction. In the remainder of the atrium, the pathway of conduction is not as strictly defined by anatomic barriers (smaller arrows).

the case of TCPC, the critical isthmus is bounded on one side by the superior and inferior venae cavae linked by a suture line and on the other side by the tricuspid anulus. The reentrant circuit exists between two barriers, the free wall TCPC suture line and the tricuspid anulus.

Implications. Successful catheter ablation of human type I AFL has been achieved. ${ }^{24,25}$ This success resulted from an understanding of the critical role of the isthmus of tissue between the tricuspid anulus and the inferior vena cava. Experience with catheter ablation of AFL after operations for congenital heart defects has been limited, and results have varied. $^{26,27} \mathrm{We}$ believe that consideration of the 
anatomy specific to each type of operation for congenital heart defects is important in the understanding potential reentrant circuits. With regard to TCPC, interruption of the corridor between the lateral tunnel suture line and the tricuspid anulus may be the key to eliminating AFL after operation.

Limitations of the study. The animal model used in this study had normal cardiac anatomy. It is possible that atrial abnormalities commonly found in children with single-ventricle physiology would result in conduction properties different from those of normal atria. The AFL circuits observed in this study were induced by pacing. It is possible that induced AFL might not be identical to tachycardias that occur spontaneously. Also, whether the AFL seen in the short term would remain inducible in the long term and whether it would involve a similar reentry circuit is unknown. Finally, by necessity, mapping of AFL took place after insertion of the intraatrial electrodes. The AFL may therefore have been affected by stretch or endocardial injury caused by these electrodes.

\section{Conclusion}

A canine model of acute AFL after the modified Fontan operation is described. The lateral tunnel suture line in TCPC construction has been demonstrated to be sufficient to create an anatomic substrate for the development and maintenance of a single distinct AFL pathway that revolves in the right atrium primarily around the venae cavae. An understanding of the anatomic constituents of AFL circuits in this model will help facilitate the future development of treatment strategies for patients undergoing the modified Fontan procedure.

We thank Donna Marquart, Tim Morris, Duane Probst, and Dennis Gordon for their expert technical assistance.

\section{REFERENCES}

1. Girod DA, Fontan F, Deville C, Ottenkamp J, Choussat A. Long-term results after the Fontan operation for tricuspid atresia. Circulation 1987;75:605-10.

2. Driscoll DJ, Offord KP, Feldt RH, Schaff HV, Puga FJ, Danielson GK. Five- to fifteen-year follow-up after Fontan operation. Circulation 1992;85:469-96.

3. Gewillig M, Wyse RK, de Leval MR, Deanfield JE. Early and late arrhythmias after the Fontan operation: predisposing factors and clinical consequences. Br Heart J 1992;67:72-9.

4. Gelatt M, Hamilton RM, McGrindle BW, et al. Risk factors for atrial tachyarrhythmias after the Fontan operation. J Am Coll Cardiol 1994:24:1735-41.

5. Puga FJ, Chiavarelli M, Hagler DJ. Modifications of the Fontan operation applicable to patients with left atrioventric- ular valve atresia or single atrioventricular valve. Circulation 1987;76(Suppl):III53-60.

6. de Leval MR, Kilner P, Gewillig M, Bull C. Total cavopulmonary connection: a logical alternative to atriopulmonary connection for complex Fontan operations. J THORAC CARDIOVASC SURG 1988;96:682-95.

7. Balaji S, Gewillig M, Bull C, de Leval MR, Deanfield JE. Arrhythmias after the Fontan procedure: comparison of total cavopulmonary connection and atriopulmonary connection. Circulation 1991;84(Suppl):III162-7.

8. Pearl JM, Laks H, Stein DG, Drinkwater DC, George BL, Williams RG. Total cavopulmonary anastomosis versus conventional modified Fontan procedure. Ann Thorac Surg 1991;52:189-96.

9. Branham BH, Bi X, Cox JL. A system for accurate interactive 3-D display of cardiac electrical activity. Institutes of Electronic and Electrical Engineers 1992;13:335-8.

10. Flinn CJ, Wolff GS, Dick M 2nd, et al. Cardiac rhythm after the Mustard operation for complete transposition of the great arteries. N Engl J Med 1984;310:1635-8.

11. Waldo AL. Atrial flutter: mechanisms, clinical features, and management. In: Zipes DP, Jalife J, eds. Cardiac electrophysiology: from cell to bedside. 2nd ed. Philadelphia: WB Saunders, 1995:666-81.

12. Klein GJ, Guiraudon GM, Sharma AD, Milstein S. Demonstration of macroreentry and feasibility of operative therapy in the common type of atrial flutter. Am J Cardiol 1986;57: 587-91.

13. Olgin JE, Kalman JM, Fitzpatrick AP, Lesh MP. Role of right atrial endocardial structures as barriers to conduction during human type atrial flutter. Circulation 1995;92:1839-48.

14. Boyden PA. Activation sequence during atrial flutter in dogs with surgically induced right atrial enlargement. I. Observations during sustained rhythms. Circ Res 1988;62:596-608.

15. Allessie MA, Bonke FI, Schopman FJ. Circus movement in rabbit atrial muscle as a mechanism of tachycardia. III. The "leading circle" concept: a new model of circus movement in cardiac tissue without the involvement of an anatomical obstacle. Circ Res 1977;41:9-18.

16. Fishberger SB, Wernovsky G, Gentles TL, Gauvreau K, Mayer JE, Walsh EP. Predictors of atrial flutter following the Fontan operation [Abstract]. J Am Coll Cardiol 1994;23: $104 \mathrm{~A}$.

17. Frame LH, Page RL, Hoffman BF. Atrial reentry around an anatomic barrier with a partially refractory excitable gap: a canine model of atrial flutter. Circ Res 1986;58:495-511.

18. Frame LH, Page RL, Boyden PA, Fenoglio JJ, Hoffman BF. Circus movement in the canine atrium around the tricuspid ring during experimental atrial flutter and during reentry in vitro. Circulation 1987;76:1155-75.

19. Schuessler RB, Boineau JB, Bromberg BI, et al. Normal and abnormal activation of the atrium. In: Zipes DP, Jalife J, eds. Cardiac electrophysiology: from cell to bedside. 2nd ed. Philadelphia: WB Saunders, 1994:549-50.

20. Rosenblueth A, Garcia Ramos J. Studies on flutter and fbrillation. II. the influence of artificial obstacles on experimental auricular flutter. Am Heart J 1947;33:677-84.

21. Boineau JP, Schuessler RB, Mooney CR, et al. Natural and evoked atrial flutter due to circus movement in dogs. Am J Cardiol 1980;45:1167-81.

22. Hayden WG, Hurley EJ, Rytand DA. The mechanism of canine atrial flutter. Circ Res 1967;20:496-505. 
23. Cronin CS, Nitta T, Mitsuno M, et al. Characterization and surgical ablation of acute atrial flutter following the Mustard procedure: a canine model. Circulation 1993;88(5 Pt 2):46171.

24. Feld GK, Fleck RP, Chen P, et al. Radiofrequency catheter ablation for the treatment of human type I atrial flutter. Circulation 1992;86:1233-40.

25. Saoudi N, Atallah G, Kirkorian G, Touboul P. Catheter ablation of the atrial myocardium in human type I atrial flutter. Circulation 1990;81:762-71.

26. Lesh MD, Van Hare GF, Epstein LM, et al. Radiofrequency catheter ablation of atrial arrhythmias: results and mechanisms. Circulation 1994;89:1074-89.

27. Triedman JK, Saul JP, Weindling SN, Walsh EP. Radiofrequency ablation of intra-atrial reentrant tachycardia after surgical palliation of congenital heart disease. Circulation 1995;91:707-14.

\section{Discussion}

Dr. Richard A. Jonas (Boston, Mass.). Dr. Rodefeld and his group invited me to comment on this presentation. I congratulate him on the presentation and congratulate the group for this fascinating study. I think the videos are absolutely spectacular.

I am not an electrophysiologist, but our group does have extensive experience with the Fontan procedure for complex single ventricle. The significance of this study is the development of an animal model that may facilitate the design of a surgical technique to minimize the incidence of inducible AFL early after a modified Fontan procedure. However, continuing work remains to be done. It is important for clinical studies to be undertaken to confirm that early inducible AFL is indeed correlated with an increased risk of late atrial arrhythmias, which is the fundamental premise and implication of this presentation.

Probably several mechanisms in addition to suture load contribute to the development of atrial reentrant tachycardia or AFL late after operations. These include the number and location of both functional and anatomic holes within the atrium, including great vein ostia and surgical incisions, the presence of abnormal atrial myocardium, and elevated atrial wall stress, which is determined by both the right atrial pressure and the diameter of the right atrium in accordance with Laplace's Law. We believe that the lateral tunnel reduces atrial wall stress by reducing the diameter of the right atrial chamber. In addition, the area of right atrial wall exposed to high pressure is minimized.

In a recent analysis of 500 patients who underwent Fontan procedures at Children's Hospital in Boston between 1973 and 1991, the following factors were found to be associated with increased risk of AFL late after operation: older age at operation, sinus node dysfunction, and nonuse of the lateral tunnel. The absence of sinus rhythm at the time of the Fontan procedure was a powerful predictor for subsequent development of AFL.

The late follow-up of our patients who have undergone the Fontan procedure suggests that the lateral tunnel approach may be associated with a reduced late incidence of atrial arrhythmias related to the use of an extracardiac conduit or an atriopulmonary anastomosis. Follow-up is too short as yet to be sure of this.

I have two questions. First, have you compared the lateral tunnel suture line with alternative suture lines, such as the oblique baffle suture line that encircled the tricuspid anulus and excised atrial septum, which we used for patients with left atrioventricular valve atresia? Clinically, this procedure appears to have been accompanied by a higher incidence of late atrial arrhythmias, and perhaps some implications of the early findings could be correlated with the late clinical findings that we already have. Second, I ask you to expand on the methods by which you would reduce the risk of AFL with the lateral tunnel approach or to speculate regarding completely novel methods that could reduce the incidence of late arrhythmias.

Dr. Rodefeld. In response to your first question, we have not looked at alternative suture line placement or baffle construction. This model was developed purely for the study of AFL after classic TCPC lateral tunnel suture line placement.

In response to your second question regarding methods to reduce the risk of AFL occurring after TCPC construction, there appear to be two potential means by which the probability of AFL can be reduced. The first, as mentioned in the presentation, is to produce a line of conduction block between the two boundaries within which the reentrant pathway conducts. This could be performed either surgically or transvenously with a cryolesion or radiofrequency ablation. The second possible alternative would be to modify the TCPC suture line in some way to try to avoid the conduction block that we have observed along the crista terminalis. If the suture line could be moved away from the crista either medially or laterally, the incidence of AFL might be reduced. Further experimental studies would be required to determine whether this would actually reduce the incidence of AFL.

Dr. Jonas. To create this block between the suture line and the tricuspid anulus, would you place a cryoprobe from the suture line inferior to the coronary sinus and just across to the tricuspid anulus?

Dr. Rodefeld. Theoretically, yes. Any nonconductive lesion between those two barriers should ablate the arrhythmia. Because the AFL pathway that we observed is narrowest in the lower right atrium, where it comes around the inferior vena cava near the tricuspid anulus, this would be a likely site for ablative therapy.

Dr. Gerhard Ziemer (Tübingen, Germany). For reasons other than avoiding possible arrhythmias-avoidance of patch leaks and to speed up the procedure by simplification-in the last 50 of our 70 patients in whom a so-called "lateral tunnel" was performed we did not create the tunnel within the right atrium with the atriotomy still open. Instead, we used the lateral patch suture line to also close the atriotomy by sandwiching the patch within the rims of the atriotomy. By this procedure, we clearly do not create any isolated lateral suture lines other than for the atriotomy.

In fact, looking at our whole series of 90 patients who have undergone the Fontan procedure and doing electrophysiologic studies on them, we discovered that about $90 \%$ of the patients with lateral tunnels chiefly con- 
structed in this way had sinus rhythm about 2 after the operation, whereas only $60 \%$ of the patients who underwent so-called more classic atriopulmonary anastomoses reveled stable sinus rhythm.

In this aspect, the lateral tunnel seems to be advantageous. After listening to your presentation, we may go back and look to see whether we can find a difference between the classic lateral tunnels and those for which, purely by accident, we may have performed an antiarrhythmic modified Fontan procedure.

Dr. Marshall L. Jacobs (Philadelphia, Pa.). I enjoyed your elegant model immensely and, like my colleague Dr. Ziemer, I found that your experiment has shed some light on what was probably a fortuitous situation with our patients in Philadelphia who underwent the Fontan procedure. Having performed more than 300 lateral tunnel TCPCs, it has routinely been the case that the medial aspect of the baffle or tunnel has been sutured in a fashion similar to what you showed in your experiments, but the lateral baffle aspect, as described by Dr. Ziemer, was incorporated directly into the closure of the atriotomy, which was routinely made approximately $1 \mathrm{~cm}$ anterior to and parallel to the crista terminalis. We have not routinely subjected these patients to electrophysiologic studies, but the incidence of spontaneous as opposed to inducible AFL in that population has anecdotally been very, very low. I was fascinated by the absence of the contribution of the atriotomy to the pathology in your model, and like Dr. Ziemer I wonder whether incorporating the suture line into the atriotomy parallel to and away from crista terminalis may represent a potential solution to this problem.

Dr. Rodefeld. I agree that this is a very interesting clinical observation, and I think that this question can really only be answered by further electrophysiologic mapping studies. It is interesting to note that injury to the crista terminalis is a common theme in many animal models of AFL, and the idea that impairment of longitudinal conduction in the crista terminalis may predispose toward reentry is intriguing and deserves further investigation.

\section{Availability of Journal back issues}

As a service to our subscribers, copies of back issues of THE Journal of THORACIC AND CARDIOVASCULAR SURGERY for the preceding 5 years are maintained and are available for purchase from the publisher, Mosby-Year Book, Inc., at a cost of $\$ 13.50$ per issue. The following quantity discounts are available: $25 \%$ off on quantities of 12 to 23 , and one third off on quantities of 24 or more. Please write to Mosby-Year Book, Inc., Subscription Services, 11830 Westline Industrial Drive, St. Louis MO 63146-3318, or call $800-453-4351$ or 314-453-4351 for information on availability of particular issues. If unavailable from the publisher, photocopies of complete issues are available from University Microfilms International, 300 N. Zeeb Rd., Ann Arbor, MI 48106, 313-761-4700. 